\title{
Comparison of the predictive performance of risk of malignancy indexes 1-4, HE4 and risk of malignancy algorithm in the triage of adnexal masses
}

Abha Hada, Li-ping Han ${ }^{*}$, Yanyan Chen, Qing-hong Hu, Yidan Yuan and Liya Liu

\begin{abstract}
Objectives: For patients presenting with adnexal mass, it is important to correctly distinguish whether the mass is benign or malignant for the purpose of precise and timely referral and implication of correct line of management. The objective of this study was to evaluate the performance of Risk of malignancy Indexes (RMI) 1-4, Human Epididymis Protein 4 (HE4) and Risk of Malignancy Algorithm (ROMA) in differentiating the adnexal mass into benign and malignant.

Methods: A retrospective study using 155 patients diagnosed with adnexal mass between January 2014 to December 2014 in The First Affiliated Hospital of Zhengzhou University was conducted. The patient records were assessed for age, menopausal status, serum CA125 and HE4 levels, ultrasound characteristics of the pelvic mass and the final pathological diagnosis of the mass. RMI1, RMI2, RMI3, RMI4, ROMA were calculated for each patient and the sensitivity, specificity and the Receiver Operating Characteristics (ROC) curves were determined for each test to evaluate their performance.
\end{abstract}

Results: Among 155 patients with adnexal masses meeting inclusion criteria, 120 (77.4\%) were benign, 8 (5.2\%) borderline and 27 (17.4\%) were malignant. RMI2 and RMI4 had the highest sensitivity (66.7\%) while HE4 had the highest specificity (96.9\%).Although ROMA had the highest area under the curve (AUC) of 0.886 it was not found to be statistically superior to the other tests. For epithelial ovarian cancers, ROMA (80\%), HE4 (96.9\%) and RMI 4 (0.868) had the highest sensitivity, specificity and AUC respectively however, the AUC characteristics were not statistically significant between any groups. Compared to the postmenopausal group (sensitivity $72.2-77.8 \%$ ) all the tests showed lower sensitivity (42.9\%) for the premenopausal group of patients.

Conclusions: RMI 1-4, ROMA and HE4 were all found to be useful for differentiating benign/borderline adnexal masses from malignant ones for deciding optimal therapy, however no test was found to be significantly better than the other. None were able to differentiate between benign and borderline tumors. All of the tests demonstrated increased sensitivity when borderline tumors were considered low-risk, and when only epithelial ovarian cancers were considered.

Keywords: Adnexal mass, Risk of malignancy index, Risk of malignancy algorithm, Human epididymis protein 4, Ovarian cancer

\footnotetext{
* Correspondence: hanliping0825@163.com

Department of Obstetrics and Gynecology, The First Affiliated Hospital of

Zhengzhou University, Zhengzhou 450002, P. R. China
}

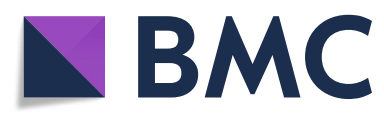

(c) The Author(s). 2020 Open Access This article is licensed under a Creative Commons Attribution 4.0 International License, which permits use, sharing, adaptation, distribution and reproduction in any medium or format, as long as you give appropriate credit to the original author(s) and the source, provide a link to the Creative Commons licence, and indicate if changes were made. The images or other third party material in this article are included in the article's Creative Commons licence, unless indicated otherwise in a credit line to the material. If material is not included in the article's Creative Commons licence and your intended use is not permitted by statutory regulation or exceeds the permitted use, you will need to obtain permission directly from the copyright holder. To view a copy of this licence, visit http://creativecommons.org/licenses/by/4.0/. The Creative Commons Public Domain Dedication waiver (http://creativecommons.org/publicdomain/zero/1.0/) applies to the data made available in this article, unless otherwise stated in a credit line to the data. 


\section{Introduction}

Ovarian cancer is the seventh most common cancer worldwide in females and the 18th most common cancer overall [1]. Its presentation with non-specific symptoms and lack of screening strategies result in delay in diagnosis which is frequently made in advanced stage, has made it a great clinical challenge among all gynecological cancers till now with the overall 5-year survival of $44 \%$ (92\% for Stage I versus $27 \%$ for Stage IV) [2]. It is crucial to differentiate between benign and malignant pelvic masses so that early and correct referral and optimal treatment can be provided, the effect of which is great on the prognosis. Furthermore, correct identification of benign the masses lessens the burden of inappropriate referral to the tertiary care centers that should focus their efforts on patients with malignancies.

Various efforts have been made to develop a system that will help to accurately differentiate a pelvic mass as benign or malignant. In 1990, Jacobs et al. developed the Risk of Malignancy Index (RMI), a risk scoring system based on menopausal status, CA125 levels and ultrasound characteristics with a sensitivity of $85.4 \%$ and a specificity of $96.9 \%$ when using a cut-off level of 200 to indicate malignancy [3]. Tingulstad et al. modified the original RMI and developed RMI2 in 1996 [4]. In 1999 the RMI 3 was developed with further modification in the scoring of ultrasound score $(\mathrm{U})$ and menopausal status (M) [5]. In 2009, Yamamoto et al. developed the RMI 4 which included tumor size (S) in the RMI [6].

Human epididymis protein 4 (HE4) is a glycoprotein first identified in the epithelium of the distal epididymis belonging to the family of whey acidic four-disulfide core (WFDC) proteins. HE4 is expressed in low amount in normal tissues including epithelia of respiratory and reproductive tissues, [7] overexpressed in $>90 \%$ of serous and endometrioid epithelial ovarian cancers and $50 \%$ of clear cell carcinomas but not in mucinous ovarian carcinomas [8]. Although its level may be increased in other malignancies such as lungs, colon and breast, highest levels are found in ovarian cancers. Moore et al. developed the Risk of Ovarian Malignancy Algorithm (ROMA), combining two biomarkers: HE4 and CA125 to categorize the patients into low risk or high risk based on their menopausal status [9].

\section{Patients and methods}

The records of all patients presenting with pelvic mass and managed in The First Affiliated Hospital of Zhengzhou University between January 2014 and December 2014 were identified. In addition to patient demographics, preoperative CA125 and HE4 levels were determined and the characterization of the mass was done by trans-abdominal and trans-vaginal ultrasonography. Patients were considered postmenopausal if they had at least 1 year of amenorrhea or for those who had undergone hysterectomy, if they were 50 years of age or older. Patients who had history of bilateral oophorectomy, ovarian cancer or any active cancer were excluded from the study. All patients underwent laparoscopic removal of the ovarian mass was and further management was decided based upon either intra operative frozen section results or final pathologic diagnosis. The final outcome was determined based on the histopathological results. Borderline tumors were categorized into the benign group for analysis purposes.

CA125 and HE4 levels were determined on cobas e 601 analyzer from Roche Diagnostics using electrochemiluminescence immunoassay (ELICA). For HE4, a cut off value of $70 \mathrm{pmol} / \mathrm{l}$ was used.

RMI calculations were made in the following manner:

RMI calculations were made using menopausal status (M), ultrasound score (U), serum CA125 value and in case of RMI 4 an additional parameter of single greatest diameter of tumor size $(\mathrm{cm}$.) (S) was included. For calculating ultrasound score, each ultrasonographic characteristics of pelvic mass: Multilocularity, solid areas, bilaterality, ascites and intra-abdominal metastases scored one point each and were summed up.

\section{RMI $1=$ M*U*CA125}

where, $M=1$ for (premenopausal)

$\mathrm{M}=3$ for postmenopausal.

$\mathrm{U}=0$ for ultrasound score of 0 .

$\mathrm{U}=1$ for ultrasound score of 1 .

$\mathrm{U}=3$ for ultrasound score of $>1$

RMI $2=M^{*} \mathrm{U}^{*} \mathrm{CA} 125$

where, $\mathrm{M}=1$ for (premenopausal)

$\mathrm{M}=4$ for postmenopausal.

$\mathrm{U}=1$ for ultrasound score of 0 or 1 .

$\mathrm{U}=4$ for ultrasound score of $>1$

\section{RMI $3=M^{*} \mathrm{U}^{*} \mathrm{CA} 125$}

where, $M=1$ for (premenopausal)

$\mathrm{M}=3$ for postmenopausal.

$\mathrm{U}=1$ for ultrasound score of 0 or 1 .

$\mathrm{U}=3$ for ultrasound score of $>1$

\section{RMI $4=M^{*} \mathrm{U}^{*} \mathrm{CA} 125^{*} \mathrm{~S}$}

where, $M=1$ for (premenopausal)

$\mathrm{M}=4$ for postmenopausal.

$\mathrm{U}=1$ for ultrasound score of 0 or 1 .

$\mathrm{U}=4$ for ultrasound score of $>1$.

$\mathrm{S}=1$ for single greatest diameter of tumor size $<7 \mathrm{~cm}$.

$\mathrm{S}=2$ for single greatest diameter of tumor size $\geq 7 \mathrm{~cm}$. 
Cut off of 200 was used for RMI 1, 2 and 3 and that of 450 was used for RMI 4.

\section{ROMA calculation:}

The algorithm was calculated using the HE4 and CA 125 values based on the menopausal status of the patient to stratify women into risk groups. A Predictive Index (PI) was calculated for premenopausal and postmenopausal patients separately using equations (1) and (2) respectively. Using Roche Elecsys specificity of $75 \%$, premenopausal women with a ROMA value $\geq 11.4$, had a higher risk of ovarian cancer. Postmenopausal women with ROMA value $\geq 29.9$ had a higher risk of ovarian cancer.

(1) Premenopausal:

$$
\mathrm{PI}=-12.0+2.38 * \mathrm{LN}[\mathrm{HE} 4]+0.0626 * \mathrm{LN}[\mathrm{CA} 125]
$$

(2) Postmenopausal:

$\mathrm{PI}=-8.09+1.04 \% \mathrm{LN}[\mathrm{HE} 4]+0.732 \% \mathrm{LN}$ [CA125] where, $\mathrm{LN}=$ Natural (Logarithm)

ROMA was calculated using the PI using following equation to calculate the predictive probability:

ROMA value $(\%)=\exp (\mathrm{PI}) /[1+\exp (\mathrm{PI})] * 100$ where, $\exp (\mathrm{PI})=\mathrm{ePI}$

Patients were classified as high risk or low risk for epithelial ovarian cancer based on following criteria.

Premenopausal women:

ROMA value $\geq 11.4 \%$ = high risk of finding epithelial ovarian cancer.

ROMA value $<11.4 \%=$ low risk of finding epithelial ovarian cancer.
Postmenopausal women:

ROMA value $\geq 29.9 \%$ high risk of finding epithelial ovarian cancer.

ROMA value $<29.9 \%=$ low risk of finding epithelial ovarian cancer.

Statistical analysis was performed using SPSS version 19. The median age of the patients was compared using Mann Whitney test, and categorical variables were compared with the Chi-square $\left(\chi^{2}\right)$ test. The Mann Whitney test was used to compare the medians of the test values in different groups. Receiver Operator characteristics (ROC) curves were constructed and the areas under the curve (AUC) for each model was compared for their accuracy. The sensitivity, specificity, positive and negative predictive values were calculated for each models using the recommended cut-off values. For all statistical comparisons, a level of $P<0.05$ was accepted as being statistically significant.

\section{Result}

Out of 155 patients who presented with pelvic mass, 113 (72.9\%) were premenopausal and 42 (27.1\%) were postmenopausal. The median age of presentation was 41 years (range 14-78). The median age in premenopausal and postmenopausal women was 32 and 53 years respectively. Among them, 120 (77.4\%) had benign, 8 (5.2\%) borderline and $27(17.4 \%)$ had malignant tumors. The median age of patients with benign, borderline and malignant tumors were 38, 37.5, 52 years respectively. The percentage of benign, borderline and malignant cases in premenopausal women were $82.5,75$ and $29.6 \%$ respectively. The percentage of benign, borderline and malignant cases in postmenopausal women were 17.5, 25 and 70.4 respectively showing that malignancy was more common in postmenopausal group $(P$-value $<$ 0.001) (Table 1).

Table 1 Baseline characteristics of the sample under study

\begin{tabular}{|c|c|c|c|c|}
\hline & & Benign & Borderline & Malignant \\
\hline Number & $\mathrm{n}(\%)$ & $120(77.4)$ & $8(5.2)$ & $27(17.4)$ \\
\hline Age (years) & Median (range) & $38(14-78)$ & $37.5(24-61)$ & $52(23-72)$ \\
\hline Postmenopausal status & $\mathrm{n}(\%)$ & $21(17.5)$ & $2(25)$ & $19(70.4)$ \\
\hline RMI1 & Median (range) & $18.03(0-1679)$ & $23.06(0-156)$ & $340.80(0-19,224)$ \\
\hline $\mathrm{RMI} 2$ & Median (range) & $35.94(4.15-1679)$ & $57.48(11.07-277.92)$ & $454.4(12.67-34,176)$ \\
\hline RMI3 & Median (range) & $31.65(4.15-1679)$ & $48.49(11.07-158.4)$ & $340.8(12.67-19,224)$ \\
\hline $\mathrm{RM} \mid 4$ & Median (range) & $52.02(4.15-3358)$ & $77.80(11.07-555.84)$ & $908.80(12.67-68,352)$ \\
\hline ROMA & Median (range) & $5.9(1.6-28.5)$ & $8.2(3.2-60.9)$ & $34.6(4-99.1)$ \\
\hline HE4 pmol/l & Median (range) & $42.17(25.37-123)$ & $47.03(34.47-163.30)$ & $57.59(35.57->1500)$ \\
\hline
\end{tabular}

RMI Risk of malignancy index, ROMA Risk Of Malignancy Algorithm, HE4 Human Epididymis Protein 4 
Table 2 Comparison of medians of the test values between benign, borderline and malignant groups

\begin{tabular}{lllllll}
\hline & RMI 1 & RMI 2 & RMI3 & RMI4 & ROMA & HE4 \\
\hline Benign vs malignant (P-value) & $<0.001$ & $<0.001$ & $<0.001$ & $<0.001$ & $<0.001$ & 0.209 \\
Benign vs borderline $(P$ - value) & 0.801 & 0.355 & 0.485 & 0.414 & 0.127 \\
Borderline vs malignant $(P$-value) & 0.002 & 0.005 & 0.005 & 0.001 & 0.013 & 0.166 \\
\hline
\end{tabular}

RMI Risk of malignancy index, ROMA Risk Of Malignancy Algorithm, HE4 Human Epididymis Protein 4

The respective median values of RMI1, RMI2, RMI3, RMI4, ROMA and HE4 were significantly different between benign and malignant group $(P$-value $<0.001)$. No significant difference was seen between the median values between benign and borderline group. Between the median values for borderline and malignant masses, significant difference was observed for all tests except HE4 (Table 2).

Among benign masses, mature teratoma was the most common (31.6\% of benign tumors) followed by functional ovarian cysts which includes corpus luteum cysts and follicular cysts and endometrioma. Among borderline tumors, borderline serous tumors was the most common $(n=5,3.5 \%)$. Among malignant tumors,

Table 3 Frequency distribution of the histopathological classification of adnexal mass

\begin{tabular}{|c|c|c|}
\hline Subtype & $\mathrm{N}$ & $\%$ \\
\hline \multicolumn{3}{|l|}{ Malignant tumors } \\
\hline Serous cystadenocarcinoma & 10 & 6.5 \\
\hline Mucinous cystadenocarcinoma & 2 & 1.3 \\
\hline Endometroid adenocarcinoma & 1 & 1.9 \\
\hline Sarcomatous tumor & 3 & 0.6 \\
\hline Immature teratoma & 2 & 1.3 \\
\hline Granulosa cell tumor (malignant) & 5 & 3.2 \\
\hline Dysgerminoma & 1 & 0.6 \\
\hline Ovarian yolk sac tumor & 1 & 0.6 \\
\hline Fallopian tube adenocarcinoma & 1 & 0.6 \\
\hline Peritoneal carcinoma & 1 & 0.6 \\
\hline \multicolumn{3}{|l|}{ Benign tumors } \\
\hline Mature teratoma & 38 & 24.5 \\
\hline Benign ovarian cysts & 32 & 20.6 \\
\hline Endometrioma & 25 & 16.1 \\
\hline Endometriod tumor & 6 & 3.9 \\
\hline Serous cystadenoma & 7 & 4.5 \\
\hline Mucinous cystadenoma & 6 & 3.9 \\
\hline Tubo-ovarian abscess & 5 & 3.2 \\
\hline Leiomyoma & 1 & 0.6 \\
\hline \multicolumn{3}{|l|}{ Borderline tumors } \\
\hline Borderline serous cystadenoma & 5 & 3.2 \\
\hline Borderline mucinous cystadenoma & 3 & 1.9 \\
\hline Total & 155 & 100 \\
\hline
\end{tabular}

epithelial ovarian cancers were the most common $(n=$ $15,9.6,56 \%$ of malignant tumors) and among them, serous cystadenocarcinomas $(n=10)$ were the most frequently observed histological subgroup. Most malignancies were stage II and above. The non-epithelial ovarian cancers were less common and consisted of 5 granulosa cell tumors, 3 sarcomatous tumors, 2 immature teratomas, 1 dysgerminoma and 1 ovarian yolk sac tumors. (Table 3).

The sensitivity, specificity, positive predictive value (PPV), negative predictive value (NPV) and Area Under the Curve of the six tests are depicted in Table 4. RMI2 and RMI 4 demonstrated the highest sensitivity whereas HE4 test demonstrated highest specificity. Comparison of the AUCs of the tests at 95\% confidence interval showed no significant difference between the tests (Fig. 1). For epithelial tumors, ROMA demonstrated the highest sensitivity compared to other tests but the AUCs of all tests at 95\% confidence intervals were comparable without significant differences as depicted in Table 5 (Fig. 2).

The sensitivity of all the six tests were lower in the premenopausal group than those in the menopausal group. In the pre- menopausal group, the HE4 had the highest AUC of 0.705 but there was no significant difference when compared to the AUCs of other tests (Fig. 3). In the post-menopausal group as well, the AUCs of the tests at $95 \%$ confidence interval did not show significant differences (Table 6, Fig. 4).

\section{Discussion}

Triage of adnexal masses presenting in the clinical setting between benign and malignant etiologies is crucial in order to guide the correct line of management. Benign masses can be managed locally whereas malignant masses are best treated in tertiary care centers by gynecologic oncologist. Ovarian cancer is often diagnosed at an advanced stage and carries a poor prognosis. Therefore characterization of the mass is important not only to determine whether a referral is needed but also to decide the line of management which has a significant impact on overall prognosis. Realizing the pressing need for the development of a system that can correctly differentiate the pelvic mass into benign or malignant, various efforts have been made and various scoring systems, marker analysis and prediction models have been developed. Despite these attempts there remains a lack of 
Table 4 Comparison of diagnostic performance of tests

\begin{tabular}{llllll}
\hline Test & Sensitivity $\%$ & Specificity $\%$ & PPV\% & NPV\% & Area under the curve (95\% confidence interval) \\
\hline RMI1 & $63(17 / 27)$ & $93.8(120 / 128)$ & $68(17 / 25)$ & $92.3(120 / 130)$ & $.844(0.740-0.947)$ \\
RMI2 & $66.7(18 / 27)$ & $89.1(114 / 128)$ & $56.3(18 / 32)$ & $92.7(114 / 123)$ & $.851(0.762-0.940)$ \\
RMI3 & $63(17 / 27)$ & $90.6(116 / 128)$ & $58.6(17 / 29)$ & $92.1(116 / 126)$ & $.841(0.749-0.932)$ \\
RMI4 & $66.7(18 / 27)$ & $92.2(118 / 128)$ & $64.3(18 / 28)$ & $92.9(118 / 127)$ & $.841(0.744-0.938)$ \\
ROMA & $59.3(16 / 27)$ & $93(119 / 128)$ & $64(16 / 25)$ & $91.5(119 / 130)$ & $.886(0.805-0.967)$ \\
HE4 & $37(10 / 27)$ & $96.9(124 / 128)$ & $71.4(10 / 14)$ & $87.9(124 / 141)$ & $.798(0.704-0.892)$
\end{tabular}

Data represented as percentages at 95\% confidence interval PPV positive predictive value, NPV negative predictive value, RMI Risk of malignancy index, ROMA Risk Of Malignancy Algorithm, HE4 Human Epididymis Protein 4. Cut off for RMI1, RMI2, RMI3 was 200, for RMI4 was 450; for ROMA cut off for premenopausal was 11.4, for postmenopausal was 29.9; for HE4 cut off of $70 \mathrm{pmol} / \mathrm{I}$ was used

universally accepted testing methodology. This study aimed to compare the performance of RMI 1, RMI 2, RMI 3, RMI 4, HE4 and ROMA in discriminating benign and malignant pelvis masses.

Risk of Malignancy Index, first published by Jacobs et al. is a scoring system based on scores from ultrasound (U), menopausal status (M), and CA-125 data in the following manner: $\mathrm{RMI}=\mathrm{U} \times \mathrm{M} \times \mathrm{CA}-125$. The original cutoff used in the study was 200 and using the sample size of 143, the sensitivity and specificity obtained were 85.4 and 96.9 respectively [3]. According to the meta -analysis conducted by Kaijser et al., the pooled summary estimates for sensitivity and specificity from 23 studies were $72 \%(67-0.76 \%$ CI) and $92 \%(89-0.93 \%$ CI) respectively [10]. RMI 2 was proposed by Tingulstad et al. in 1996 modifying the scores assigned to the ultrasound and menopause components of RMI 1 using the cutoff level of 200. The original study include 173 patients and the sensitivity and specificity obtained were 71 and $96 \%$ respectively. The pooled summary estimates for sensitivity and specificity from 15 studies were found to be $75 \%(69-80 \% \mathrm{CI})$ and $87 \%(84-90 \% \mathrm{CI})$ [10].

In 1999, Tingulstad et al. further modified the two previous RMI models, and introduced RMI 3 assigning new scores to the ultrasound and menopause components using the same cut off level. The original study included 365 patients with obtained sensitivity and specificity of 71 and $92 \%$ respectively [5]. The pool summary of 9 studies conducted reflects the sensitivity of $70 \%(60-78 \% \mathrm{CI})$ and specificity of 91\% (88-93\% CI) [10]. In 2009, Yamamoto et al. developed the RMI 4 which included tumor size (S) as an additional component to previous versions of RMIs using a cutoff level of 450. In their retrospective study with 253 cases RMI 4 was found to have sensitivity

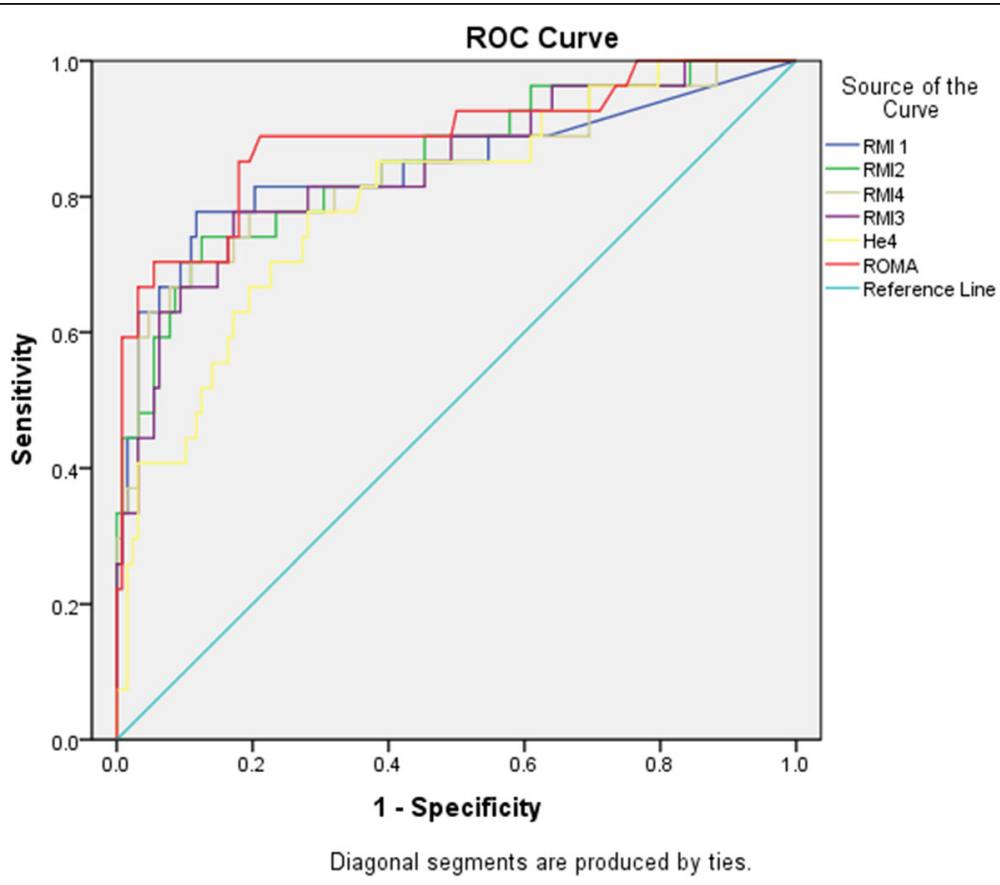

Fig. 1 ROC curve benign vs all ovarian cancers 
Table 5 Comparison of diagnostic performance of tests for epithelial ovarian cancers

\begin{tabular}{|c|c|c|c|}
\hline Test & Sensitivity\% & Specificity\% & Area under the curve (95\% confidence interval) \\
\hline RMI1 & 73.3 & 93.8 & $0.861(0.731-0.992)$ \\
\hline RMI2 & 73.3 & 89.1 & $0.847(0.715-0.979)$ \\
\hline RMI3 & 73.3 & 90.6 & $0.861(0.731-0.992)$ \\
\hline RMI4 & 73.3 & 92.2 & $0.868(0.742-0.994)$ \\
\hline ROMA & 80 & 93 & $0.861(0.731-0.992)$ \\
\hline HE4 & 53.3 & 96.9 & $0.653(0.479-0.827)$ \\
\hline \multicolumn{4}{|c|}{ Pre-menopausal } \\
\hline RMI 1 & $42.9 \%$ & $91.5 \%$ & $0.672(0.432-0.912)$ \\
\hline RMI 2 & $42.9 \%$ & $87.7 \%$ & $0.653(0.416-0.890)$ \\
\hline RMI 3 & $42.9 \%$ & $87.7 \%$ & $0.653(0.416-0.890)$ \\
\hline RMI 4 & $42.9 \%$ & $90.6 \%$ & $0.667(0.428-0.907)$ \\
\hline HE4 & $42.9 \%$ & $98.1 \%$ & $0.705(0.459-0.950)$ \\
\hline ROMA & $42.9 \%$ & $91.5 \%$ & $0.672(0.432-0.912)$ \\
\hline \multicolumn{4}{|c|}{ Menopausal } \\
\hline RMI 1 & $72.2 \%$ & $100 \%$ & $0.861(0.731-0.992)$ \\
\hline RMI 2 & $77.8 \%$ & $91.7 \%$ & $0.847(0.715-0.979)$ \\
\hline RMI 3 & $72.2 \%$ & $100 \%$ & $0.861(0.731-0.992)$ \\
\hline RMI 4 & $77.8 \%$ & $95.8 \%$ & $0.868(0.742-0.994)$ \\
\hline HE4 & $77.8 \%$ & $91.7 \%$ & $0.653(0.479-0.827)$ \\
\hline ROMA & $77.2 \%$ & $100 \%$ & $0.861(0.731-0.992)$ \\
\hline
\end{tabular}

Data represented as percentages at $95 \%$ confidence interval

PPV positive predictive value, NPV negative predictive value, RMI Risk of malignancy index, ROMA Risk Of Malignancy Algorithm, HE4 Human Epididymis Protein 4. Cut off for RMI1, RMI2, RMI3 was 200, for RMI4 was 450; for ROMA cut off for premenopausal was 11.4, for postmenopausal was 29.9 ; for HE4 cut off of 70 pmol/l was used



Diagonal segments are produced by ties.

Fig. 2 ROC curve benign vs epithelial ovarian cancers 
Table 6 Comparison of performance of tests in premenopausal and postmenopausal groups

\begin{tabular}{llll}
\hline & Sensitivity & Specificity & Area Under the Curve (95\% confidence interval) \\
\hline Pre-menopausal & & & \\
RMI 1 & $42.9 \%$ & $91.5 \%$ & $0.672(0.432-0.912)$ \\
RMI 2 & $42.9 \%$ & $87.7 \%$ & $0.653(0.416-0.890)$ \\
RMI 3 & $42.9 \%$ & $87.7 \%$ & $0.653(0.416-0.890)$ \\
RMI 4 & $42.9 \%$ & $90.6 \%$ & $0.667(0.428-0.907)$ \\
HE4 & $42.9 \%$ & $98.1 \%$ & $0.705(0.459-0.950)$ \\
ROMA & $42.9 \%$ & $91.5 \%$ & $0.672(0.432-0.912)$ \\
Menopausal & & & \\
RMI 1 & $72.2 \%$ & $100 \%$ & $0.861(0.731-0.992)$ \\
RMI 2 & $77.8 \%$ & $91.7 \%$ & $0.847(0.715-0.979)$ \\
RMI 3 & $72.2 \%$ & $100 \%$ & $0.861(0.731-0.992)$ \\
RMI 4 & $77.8 \%$ & $95.8 \%$ & $0.868(0.742-0.994)$ \\
HE4 & $77.8 \%$ & $91.7 \%$ & $0.653(0.479-0.827)$ \\
ROMA & $77.2 \%$ & $100 \%$ & $0.861(0.731-0.992)$ \\
\hline
\end{tabular}

RMI Risk of malignancy index, ROMA Risk Of Malignancy Algorithm, HE4 Human Epididymis Protein 4

and specificity of 86.8 and 91 respectively. The pool summary of 3 studies conducted reflects the sensitivity of $68 \%$ (59-76\% CI) and specificity of 94\% (91-96\% CI) [10].

The serum biomarker HE4 was introduced as a novel and promising marker by Hellstrom et al. [11] and in 2008 has been cleared by the U.S. Food and Drug Administration for ovarian cancer monitoring. Moore et al. in 2009 introduced a biomarker based algorithm based the combination of two pilot studies, ROMA which combines the results of HE4, CA125 and menopausal status to calculate a risk score and categorizes the mass as high risk or low risk for malignancy. It was then subsequently validated in a multicenter trial assessing women presenting with pelvic mass. While evaluating 531 pelvic mass patients, the sensitivity for benign vs malignant was found to be $93.8 \%$ at specificity of $75 \%$; $88.9 \%$ for premenopausal and $94.5 \%$ for post menopausal women at a fixed specificity of $75 \%[12,13]$.

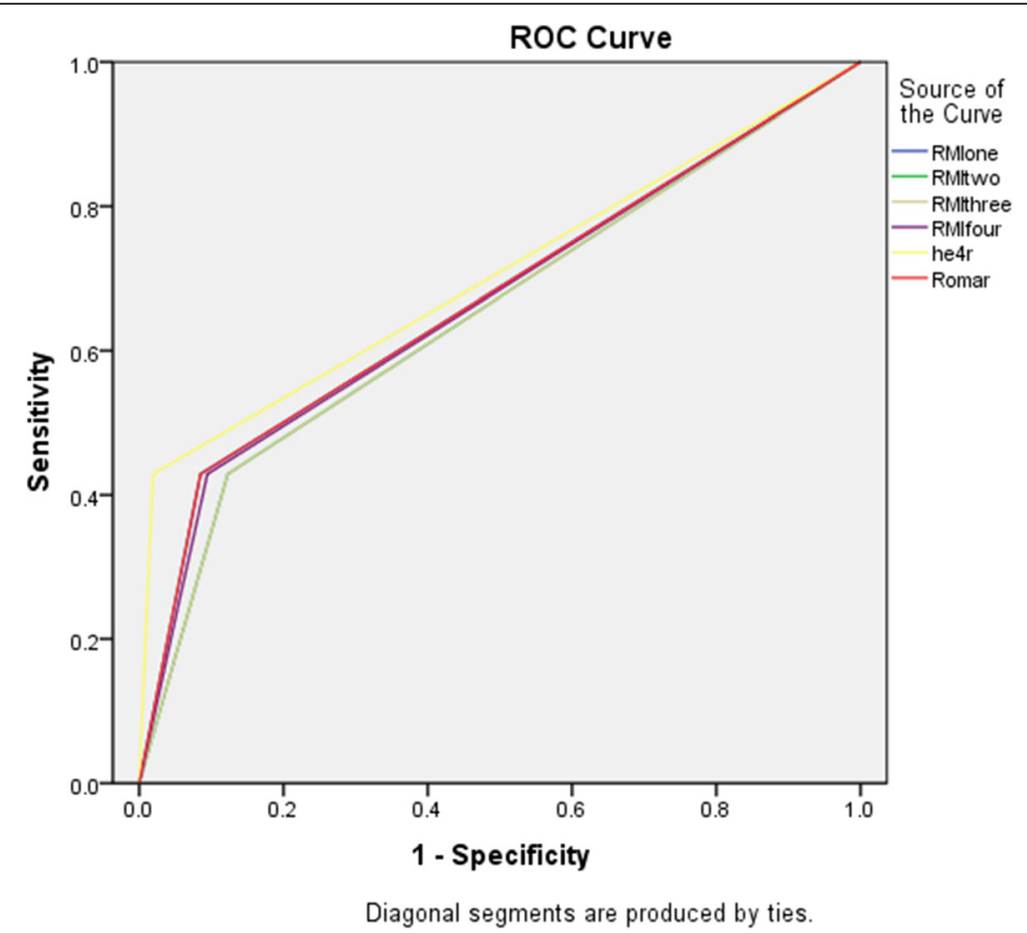

Fig. 3 ROC curve benign vs all ovarian cancers in premenopausal 


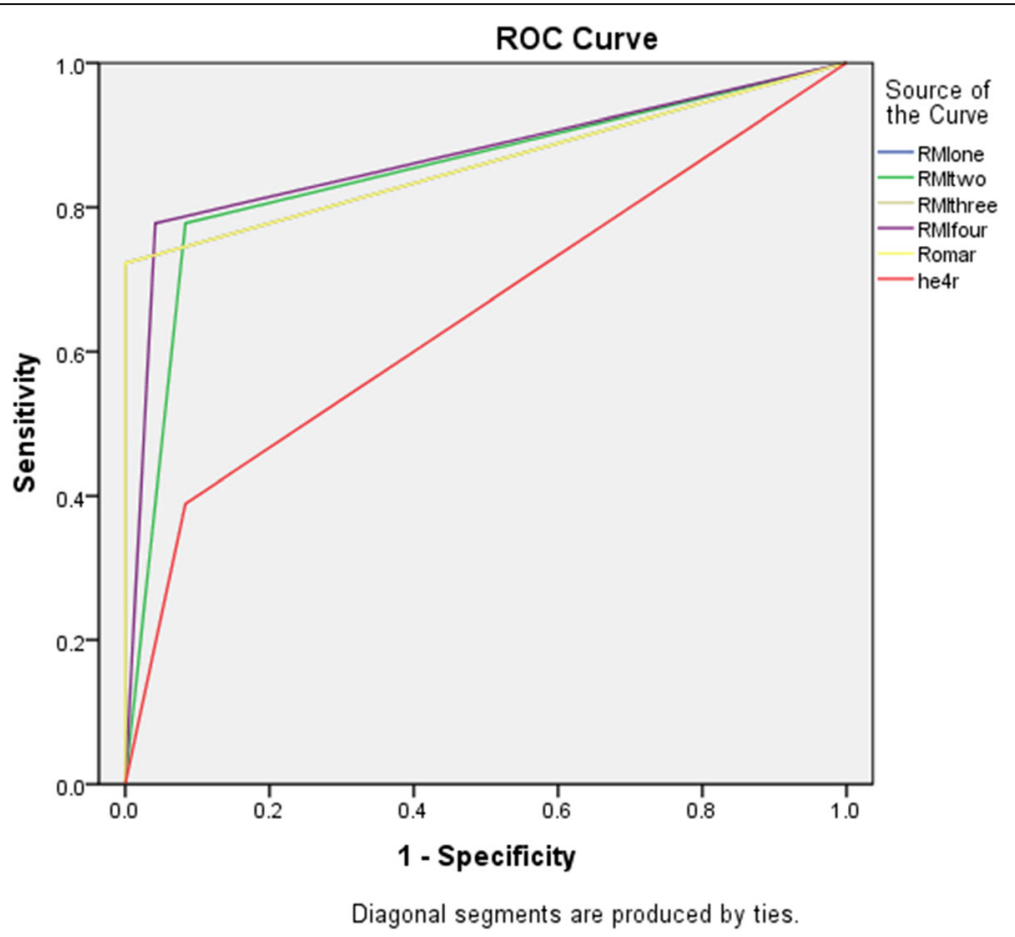

Fig. 4 ROC curve benign vs all ovarian cancers for postmenopausal

This study was conducted to compare the performance of each test for the same set of cases presenting with pelvic adnexal mass. In this study it was found that RMI 1, RMI2, RMI3, RMI4, HE4 and ROMA were all able to differentiate between benign and malignant masses. The borderline tumors when considered in the benign group, higher sensitivity were obtained for each tests. The sensitivity, specificity and AUC for the tests when overall tumors were considered were 63, 93.8\% and 0.844 (RMI1); 66.7, 89.1\% and 0.851 (RMI2); 63, 90.6\% and 0.841 (RMI3); 66.7, 92.2\% and 0.841 (RMI4); 59.3, $93 \%$ and 0.886 (ROMA) and $37,96.9 \%$ and 0.798 (HE4) respectively.. The difference in AUC between the tests were not found to be significant. When median values of the test for benign, borderline and malignant tumors were compared, no test was found to have significantly different value for benign and borderline tumors and all except for HE4 had significantly different median values for borderline and malignant tumors. When only epithelial ovarian cancers were considered, it was found that the sensitivity of each test was higher compared to that when overall tumors were considered: 73.3\% (RMI 1), 73.3\% (RMI2, RMI3, RMI4), 80\% (ROMA) and 53.3\% (HE4). The ROMA had the highest sensitivity in that case but the AUC between different tests were not found to be significantly different. The sensitivity of the tests were found to be lower in the premenopausal group than that in postmenopausal group.

\section{Conclusion}

RMI 1-4, ROMA and HE4 were all found to be useful for differentiating benign/borderline adnexal masses from malignant ones for deciding optimal therapy, however no test was found to be significantly better than the other. None were able to differentiate between benign and borderline tumors. All of the tests demonstrated increased sensitivity when borderline tumors were considered low-risk, and when only epithelial ovarian cancers were considered.

\section{Abbreviations \\ RMI: Risk of Malignancy Index; ROMA: Risk Of Malignancy Algorithm; HE4: Human Epididymis Protein 4; PPV: Positive Predictive Value; NPV: Negative Predictive Value; AUC: Area Under the Curve; ROC: Receiver Operator Characteristics}

\section{Acknowledgements \\ Not Applicable.}

\section{Authors' contributions}

HA is the primary author of this manuscript, put forward the concept of the study, involved in data collection, analysis and interpretation. LH contributed to data interpretation and provided valuable inputs to modify the manuscript. $\mathrm{CY}$ and $\mathrm{HQ}$ assisted in data collection and translation of the data from Chinese to English. LL and YY assisted in manuscript revision and editing. All authors read and approved the final manuscript.

\section{Funding}

This study was supported by the National Natural Science Foundation of China (No. U1604172), the Science and Technology Planning Project of Henan Province co-established by the province and the ministry (No. 201701002) and the Science and Technology Colleges Innovation Team Support Program of Henan Province (No. 18IRTSTHN024). 


\section{Availability of data and materials}

The datasets generated during and/or analysed during the current study are available from the corresponding author on reasonable request.

\section{Ethics approval and consent to participate}

Not Applicable.

\section{Consent for publication}

Not Applicable.

\section{Competing interests}

The authors declare that they have no competing interests.

Received: 10 October 2019 Accepted: 3 April 2020

Published online: 25 April 2020

\section{References}

1. Ferlay J, Soerjomataram I, Ervik M, et al. GLOBOCAN 2012 v1.0, Cancer incidence and mortality worldwide: IARC CancerBase no. 11. Lyon: International Agency for Research on Cancer; 2013. Available from: http:// globocan.iarc.fr, accessed Dec 2013

2. Siegel R, Ma J, Zou Z, Jemal A. Cancer statistics, 2014. CA Cancer J Clin. 2014;64(1):9-29.

3. Jacobs I, Oram D, Fairbanks J, Turner J, Frost C, Grudzinskas JG. A risk of malignancy index incorporating CA-125, ultrasound and menopausal status for the accurate preoperative diagnosis of ovarian cancer. Br J Obstet Gynecol. 1990;97:922-9.

4. Tingulstad S, Hagen B, Skjeldestad FE, et al. Evaluation of risk of malignancy index based on serum CA 125, ultrasound findings and menopausal status in the preoperative diagnosis of pelvic masses. Br J Obstet Gynecol. 1996; 103:826-31.

5. Tingulstad S, Hagen B, Skjeldestad FE, Halvorsen T, Nustad K, Onsrud M. The risk of malignancy index to evaluate potential ovarian cancers in local hospitals. Br J Obstet Gynecol. 1999;93:448-52.

6. Yamamoto Y, Yamada R, Oguri H, Maeda N, Fukaya T. Comparison of four malignancy risk indices in the preoperative evaluation of patients with pelvic masses. Eur J Obstet Gynaecol Reprod Biol. 2009;144:163-7.

7. Galgano MT, Hampton GM, Frierson HF Jr. Comprehensive analysis of HE4 expression in normal and malignant human tissues. Mod Pathol. 2006;19: 847-53.

8. Drapkin R, von Horsten HH, Lin Y, Mok SC, Crum CP, Welch WR, Hecht JL. Human epididymis protein 4 (HE4) is a secreted glycoprotein that is overexpressed by serous and endometrioid ovarian carcinomas. Cancer Res. 2005:65:2162-9.

9. Moore RG, Jabre-RaughleyM BAK, Robison KM, Miller MC, Allard WJ, et al. Comparison of a novelmultiplemarker assay vs the risk of malignancy index for the prediction of epithelial ovarian cancer in patients with a pelvic mass. Am J Obstet Gynecol. 2010;203:226-8.

10. Kaijser J, Sayasneh A, Van Hoorde K, Ghaem-Maghami S, Bourne T, Timmerman D, et al. Presurgical diagnosis of adnexal tumours using mathematical models and scoring systems: a systematic review and metaanalysis. Hum Reprod Update. 2014;20(3):449-62.

11. Helistrom I, Raycraft J, Hayden-Ledbetter M, Ledbetter JA, Schummer M, Mclntosh M, Drescher C, Urban N, Hellstrom KE. The HE4 (WFDC2) protein is a biomarker for ovarian carcinoma. Cancer Res. 2003;63:3695-700.

12. Moore RG, MCMeekin DS, Brown AK, DiSilvestro P, Miller MC, Allard WJ, et al. A novel multiple marker bioassay utilizing HE4 and CA125 for the prediction of ovarian cancer in patients with a pelvic mass. Gynecol Oncol. 2009;112: 40-6.

13. Moore RG, Brown AK, Miller MC, Skates S, Allard WJ, Verch T, et al. The use of multiple novel tumor biomarkers for the detection of ovarian carcinoma in patients with a pelvic mass. Gynecol Oncol. 2008;108:402-8.

\section{Publisher's Note}

Springer Nature remains neutral with regard to jurisdictional claims in published maps and institutional affiliations.

\section{Ready to submit your research? Choose BMC and benefit from:}

- fast, convenient online submission

- thorough peer review by experienced researchers in your field

- rapid publication on acceptance

- support for research data, including large and complex data types

- gold Open Access which fosters wider collaboration and increased citations

- maximum visibility for your research: over $100 \mathrm{M}$ website views per year

At BMC, research is always in progress.

Learn more biomedcentral.com/submissions 